\title{
Hilbert transform revisited - Proper orthogonal decomposition applied to analytical signals of flow fields
}

\author{
Jochen Kriegseis $^{1 *}$, Matthias Kinzel ${ }^{2}$, Holger Nobach ${ }^{3}$ \\ ${ }^{1}$ Institute of Fluids Mechanics (ISTM), Karlsruhe Institute of Technology (KIT), Karlsruhe, Germany \\ ${ }^{2}$ BlackSky, Spaceflight Industries, Seattle, WA, USA \\ ${ }^{3}$ Max Planck Institute for Dynamics and Self-Organization, Göttingen, Germany \\ *kriegseis@kit.edu
}

\begin{abstract}
The modes delivered by proper orthogonal decomposition (POD) are uncorrelated as per definition; but interestingly, they are not necessarily independent in terms of spatio-temporal flow-pattern dynamics. For instance, periodic structures that travel as waves through a series of snapshots often consist of pairs of modes with harmonic functions shifted 90 degree in phase and/or a spatial offset by a quarter of the spatial wave length of the convective flow pattern. Identification of such pairs, however, largely builds upon experience, visual inspection and/or the analysis of the reconstructed coefficients in cyclograms (Lissajous figures). This effort becomes even more challenging if measurement noise or other spurious information contaminates the raw data under consideration. One possibility to automatically pair corresponding patterns with common POD algorithms is the immediate application of the POD method to complex data (see Pfeffer et al., 1990). As outlined by Horel (1984), the Hilbert transform is a well-known and straight forward means to obtain the required extension of the original signal with an appropriate 90 degrees phase shift, which is independent of the fundamental frequencies. The complex extension of the original (real) signal $X_{i}$ and its (discrete) Hilbert transform $\operatorname{HT}\left\{X_{i}\right\}$ as the imaginary part $X_{i}+\mathbf{i} \operatorname{HT}\left\{X_{i}\right\}$ with the imaginary unit $\mathbf{i}$ is commonly known as the so-called analytical signal.

When applied to a given data set as a preparation step to a subsequent snapshot POD analysis (Sirovich, 1987), each property of the data at hand can be converted into a complex analytical signal in time. The complex extension based on the Hilbert transform then applies along the snapshots direction for each point in space and for each component per spatial location individually. Since the Hilbert transform is the only modification of the original data which then undergoes the complex, but otherwise usual snapshot POD analysis, the proposed method will be abbreviated and referred to as HPOD below. The results of the HPOD are comprised of real eigenvalues and corresponding complex modes. Note that the analytical signal obtained by the Hilbert transform cannot increase the information content of the input data, i.e. the original data and the complex extended data have identical information. Therefore, also the result of the modal decomposition by HPOD contains equivalent information. However, the complex modes can combine the pairs of coupled modes to single complex modes. These complex modes, therefore, uniquely capture amplitude and phase of both spatial and temporal evolution of moving flow patterns (Barnett, 1983), which can also uncover the phase drift for traveling wave structures, for instance. The major advantage, consequently, is a straight forward interpretation of the different modes, which moreover becomes largely independent of expertise and subjectivity. Interestingly enough, the HPOD approach remains as yet only rarely applied in fluid mechanics - despite these obvious advantages.

The purpose of the present work, therefore, centers around a thorough elaboration of the HPOD capabilities for an advanced analysis of flow-field information. In order to evaluate both advantages and limits of the HPOD approach, three different tow-dimensional (2D) test cases are chosen, each of which is known to be dominated by periodic (oscillatory) convection of flow patterns. To demonstrate the independence of the analytical signal from the number of considered properties per spatial location, single-, two- and three component flow fields $(1 \mathrm{C}, 2 \mathrm{C}, 3 \mathrm{C})$ have been considered - random snap shots of all three test cases are shown in Figure 1 for introductory purposes. To furthermore mimic temporally undersampled data, arbitrarily ordered snap shots have also been applied to the Hilbert transform prior to the subsequent HPOD application.
\end{abstract}




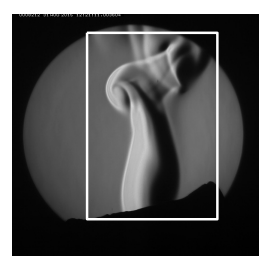

(a) burning candle

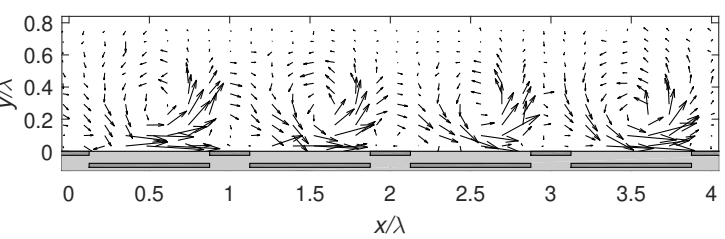

(b) discharge-based Stokes layer

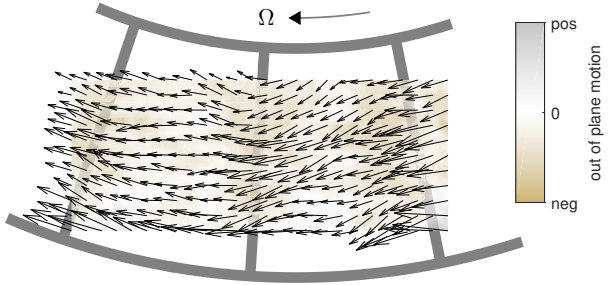

(c) side-channel flow

Figure 1: Random snapshots of the measured flow fields under consideration: (a) 2D1C data: Schlieren images in proximity of a flickering candle (data from Laier, 2015), the white box indicates the range of interest (ROI) for the present study; (b) 2D2C data: phase-resolved planar PIV fields above an oscillatory operating plasma-actuator array (data from Hehner et al., 2019); (c) 2D3C data: time-resolved stereo PIV data in the side channel of a regenerative pump (data from Mattern et al., 2017)

The comparison of HPOD results with standard POD successfully demonstrates that the desired automatic identification of pairs of corresponding patterns is possible when applied to the analytical signal of the raw data, where two related POD modes are combined in a single complex HPOD mode. The histories of reconstructed coefficients furthermore suggest that the analytical signal of the Hilbert-transformed raw data keeps it's helical character across intensity fluctuations, frequency sweep and spurious noise contributions of the respective dynamics. This insight holds for single- and multi-component data sets and does not suffer from low signal-to-noise ratios. Furthermore, the artificially undersampled data sets lead to arbitrary imaginary patterns, yet preserve the salient modal patterns in the real part of the eigenvectors. This insight leads to the conclusion that an HPOD application to undersampled data effectively only reduced to quasi-standard POD results, but still reveals meaningful information in the classical POD sense.

As a final remark, the above insights and conclusions indicate that the Hilbert-transform based conversion of raw data to an analytical signal is an advantageous additional robust and straight forward preprocessing step to advance beyond the classical POD method. The simplicity of this modification renders the HPOD a promising decomposition option for periodical and/or fluctuating flow scenarios.

\section{References}

Barnett TP (1983) Interaction of the Monsoon and Pacific Trade Wind System at Interannual Time Scales Part I: The Equatorial Zone. Monthly Weather Review 111:756-773

Hehner MT, Gatti D, and Kriegseis J (2019) Stokes-layer formation under absence of moving parts-a novel oscillatory plasma actuator design for turbulent drag reduction. Physics of Fluids 31:051701

Horel JD (1984) Complex Principal Component Analysis: Theory and Examples. Journal of Climate and Applied Meteorology 23:1660-1673

Laier M (2015) Schlieren investigations into weak density gradients. Bachelor's Project, Institute of Fluid Mechanics, Karlsruhe Institute of Technology

Mattern P, Gabi M, and Kriegseis J (2017) A plane-to-plane comparison of common averaged vs. POD patterns of time-resolved stereo-PIV data within a pump. European Journal of Mechanics-B/Fluids 61:321329

Pfeffer RL, Ahlquist J, Kung R, Chang Y, and Li G (1990) A Study of Baroclinic Wave Behavior over Bottom Topography Using Complex Principal Component Analysis of Experimental Data. Journal of the Atmospheric Sciences 47:67-81

Sirovich L (1987) Turbulence and the dynamics of coherent structures part I: Coherent structures. Quarterly of Applied Mathematics 45:561-571 